\title{
Proton irradiation of malignant melanoma of the ciliary body
}

\author{
EVANGELOS S. GRAGOUdAS, ${ }^{1}$ MICHAEL GOITEIN, ${ }^{2}$ ANDREAS KOEHLER, ${ }^{3}$ \\ MILES S. WAGNER, ${ }^{3}$ LYNN VERHEY, JOEL TEPPER, ${ }^{2}$ HERMAN D. SUIT, ${ }^{2}$ \\ ROBERT J. SCHNEIDER, ${ }^{3}$ AND KRISTEN N. JOHNSON, ${ }^{3}$ \\ From the ${ }^{1}$ Eye Research Institute of Retina Foundation and Massachusetts Eye and Ear Infirmary, and the \\ ${ }^{2}$ Department of Radiation Medicine, Massachusetts General Hospital, Boston, Massachusetts, \\ and ${ }^{3}$ Harvard Cyclotron Laboratory, Cambridge, Massachusetts
}

SUMMARY This is our first case of malignant melanoma of the ciliary body treated with proton beam irradiation, a technique that we developed for irradiating choroidal melanomas. After 21 months of follow-up no growth of the tumour has been observed, and shrinkage of the tumour was noted on the follow-up photographs and by ultrasonography. The ${ }^{32} \mathrm{P}$ uptake test, which was positive before treatment, turned negative 14 months after irradiation. The described technique of proton beam irradiation might offer an alternative for the treatment of ciliary body melanomas when the present techniques of iridocyclectomy cannot be applied because of the size of the lesion.

During recent years local excision of certain ciliary body melanomas has provided an attractive alternative to enucleation (Stallard, 1964; Muller et al., 1964; Palm and Linder, 1968; Linnic, 1968; Reese et al., 1968; and Vail, 1971). Unfortunately this technique can be applied only to relatively small tumours. A lesion occupying more than 2.5 clock hours usually cannot be safely excised (Muller et al., 1966).

Local irradiation with cobalt- 60 plaques has also been used as an alternative treatment but generally has been considered unsatisfactory, and enucleation has often been necessary owing to postirradiation complications (Stallard, 1973). During the last 2 years we have used a high-energy proton beam for the treatment of choroidal melanomas because of the multiple advantages provided by this technique for localising ocular irradiation (Gragoudas et al., 1977). The accuracy and reliability of this method encouraged us to use it for irradiation of those ciliary body melanomas that are considered inoperable with the present techniques of iridocyclectomy. This report presents our first case of malignant melanoma of the ciliary body treated with proton beam irradiation.

\section{Case report}

A 57-year-old white woman was examined because of a slight decrease in vision in her left eye over

Address for reprints: Editorial Services Unit, Eye Research Institute of Retina Foundation, 20 Staniford Street, Boston, Massachusetts 02114, USA the past 6 months. Visual acuity was $6 / 7.5$ in the right eye and 6/12 in the left. Slit-lamp biomicroscopy revealed a protruding mass at the inferior portion of the iris, which was slightly atrophic and hyperpigmented in that area (Fig. 1). Gonioscopy showed that the iris was 'tented up' inferiorly, obscuring part of the angle. After dilatation of the pupil, a raised, lobulated, pigmented mass of the ciliary body was detected, extending from the 5 to the 8 o'clock position. The mass, which pressed against the periphery of the lens inferiorly, had produced cataractous changes (Figs. 2, 3). The view of the inferior retina was not clear owing to the lens changes, but transillumination revealed no extension of the mass beyond the pars plana. Fluorescein angiography using a Goldmann three-mirror lens showed some leakage of the vessels over the tumour, and measurements of the uptake of radioactive phosphorus $\left({ }^{32} \mathrm{P}\right)$ showed a $61 \%$ greater uptake over the tumour than in the same area of the fellow eye. The dimensions of the tumour, calculated from ultrasound measurements and direct measurements obtained during transillumination, were $8 \times 6.5 \times$ $3 \mathrm{~mm}$. Owing to the large size of the lesion, iridocyclectomy was deemed very difficult, and treatment by proton irradiation was advised.

\section{Design of proton irradiation field and treatment plan}

Since the lesion was in the anterior half of the eye and relatively superficial, it lent itself well to an irradiation en face with a single proton beam. The 


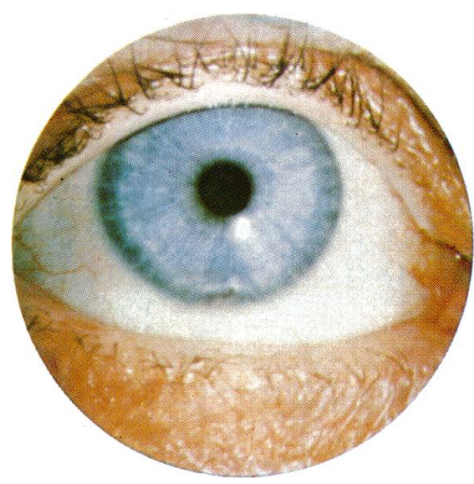

Fig. 1 Atrophy of the iris at the 6-o'clock position, surrounded by hyperpigmentation

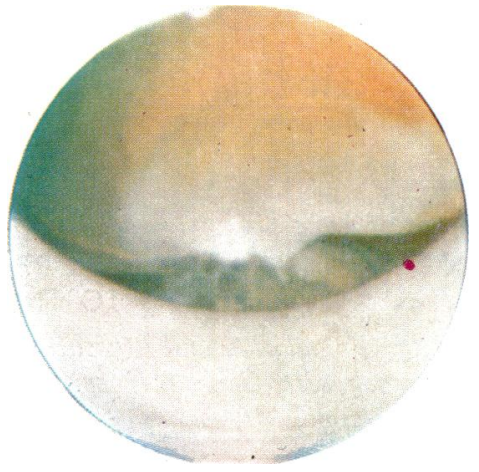

Fig. 2 Lobulated pigmented mass pressing against the periphery of the lens, producing localised cataract, as seen after pupillary dilatation

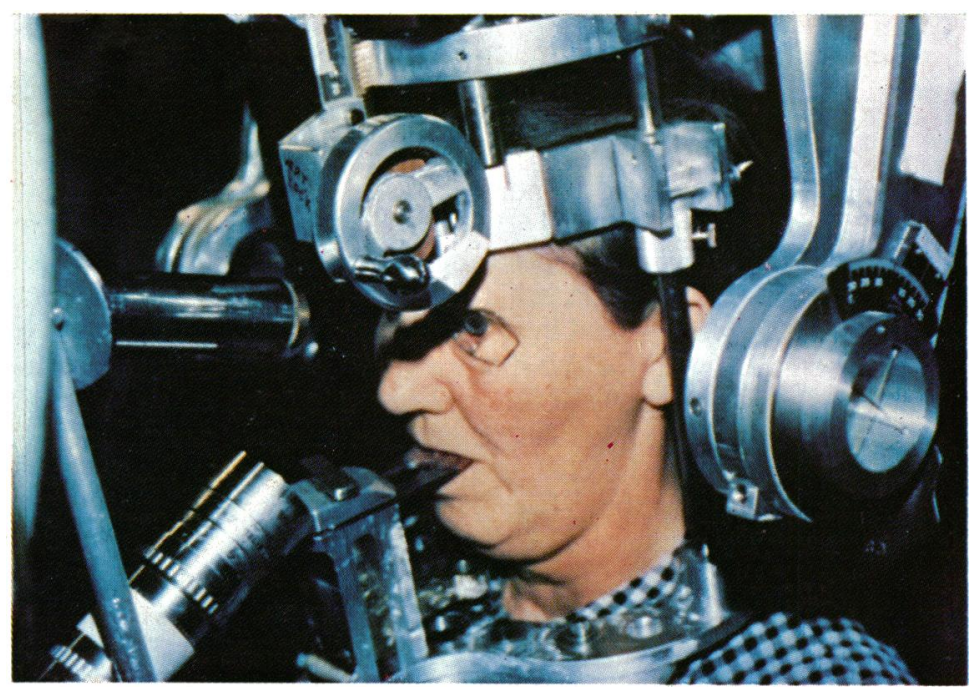

Fig. 6 Patient in the headholder, looking at the fixation light.

Television camera provides magnified image of patient's eye in the control area

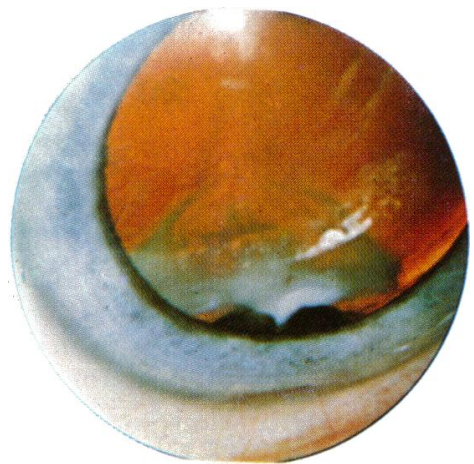

Fig. 7 Lesion 8 months after irradiation

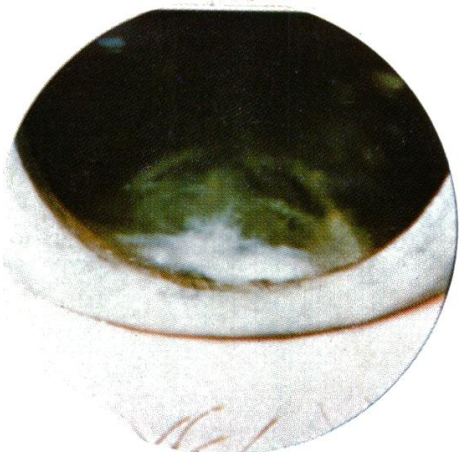

Fig. 8 Lesion 18 months after irradiation

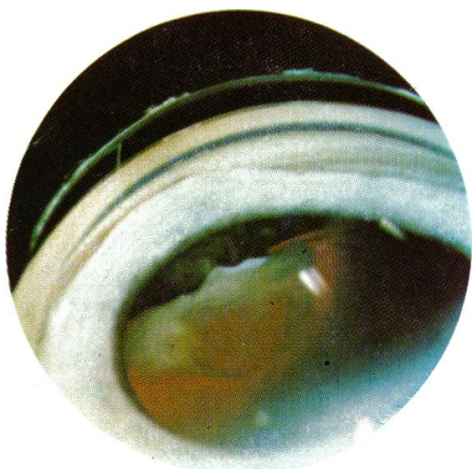

Fig. 3 Lesion, lens changes, and elevated iris as seen on gonioscopy after pupillary dilatation 
patient's head was rotated downward and her gaze directed upward in order to bring the lesion clear of the lower eyelid and to allow the proton beam, which is constrained to the horizontal direction, to be more closely perpendicular to the tumour. The proton penetration was selected so that the distal $90 \%$ isodose would be achieved $5 \mathrm{~mm}$ from the surface of the eye. Since the lesion was superficial, a single Bragg peak was sufficient to assure uniform dose distribution throughout the tumour volume, and modulation of the depth of penetration, which is commonly employed in our practice (Gragoudas et al., 1977), was not necessary.

We are able to design fields of almost any crosssection by fabrication of appropriate apertures (Gragoudas et al., 1977). In the case of this patient a circular beam seemed highly appropriate. Because the angle between the proton beam and the axis of the eye was approximately $23^{\circ}$, the shape of the beam projected on to the plane containing the lens and ciliary body was slightly elliptical. Fig. 4 indicates the 20,50 , and $90 \%$ isodose curves in this plane. The dose distribution achieved in a sagittal plane is indicated in Fig. 5. in which the same isodose levels are shown. The treatment volume is essentially that enclosed by the line labelled ' 90 ' in these figures. In the design of the field, margins of $2 \mathrm{~mm}$ superiorly and inferiorly and $3 \mathrm{~mm}$ laterally were allowed

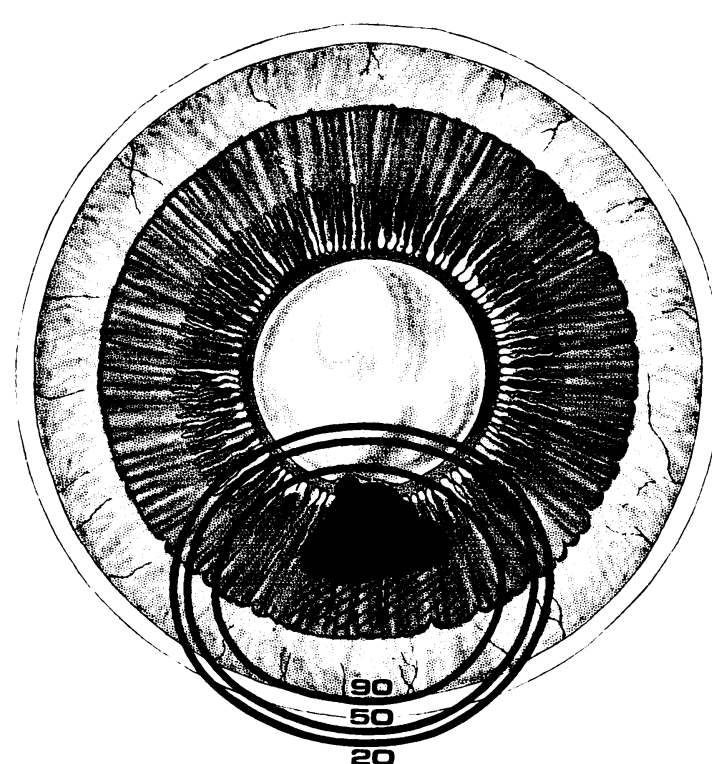

Fig. 4 Isodose lines demonstrating the irradiated ocular tissues with 20,50, and $90 \%$ of the total dose. Diagram shows ciliary body and tumour; black part represents gross tumour, and diagonal lines indicate area of possible tumour extension

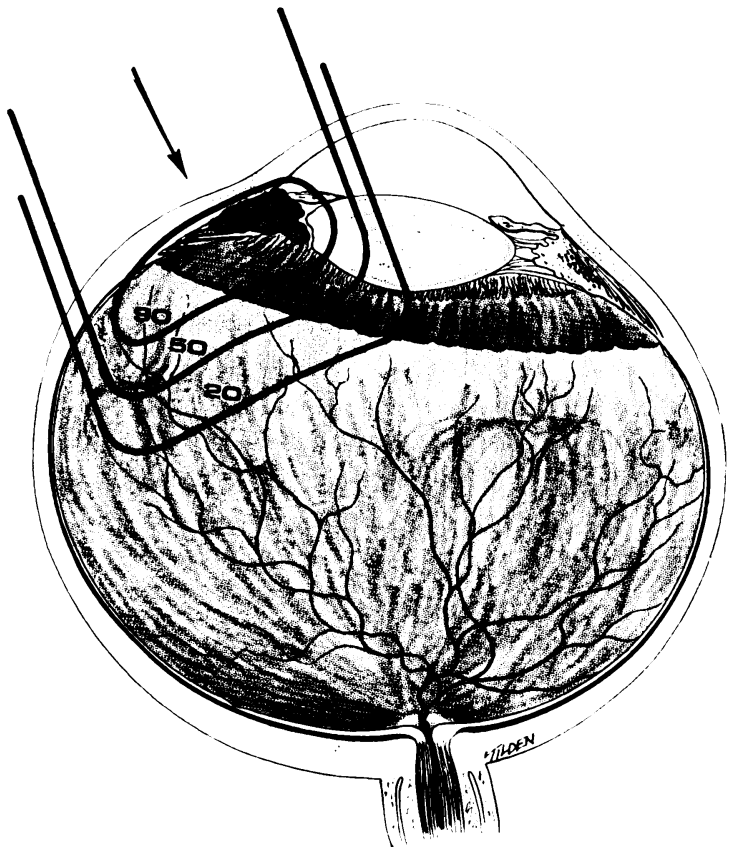

Fig. 5 Dose distribution curves shown in a sagittal plane

between the tumour and the $90 \%$ isodose curves to allow for microscopic extension of the lesion, motion during treatment, and alignment error. This margin is greater than that conventionally used by us in the treatment of choroidal melanomas, where we are able to take advantage of radiopaque markers that are precisely located relative to the macroscopically visible tumour (Gragoudas et al., 1977). In irradiating ciliary body lesion the alignment procedure was guided by visual anatomical landmarks located in the iris and conjunctiva and defined on the ocular surface previously by transillumination.

\section{PROTON BEAM COLLIMATION AND} DOSIMETRY

The $160-\mathrm{MeV}$ proton beam generated by the Harvard cyclotron has been described in detail previously (Kjellberg and Klinian, 1975). Briefly, an attenuator is placed in the path of the beam, both degrading the energy to achieve the desired penetration within the eye and scattering the pencil beam to achieve uniform irradiation over the field. The protons are then permitted a flight path of approximately $90 \mathrm{~cm}$, during which secondary collimation reduces the possibility of scattered protons reaching the patient. Dual transmission ionisation chambers monitor the beam during treatment, and the final collimation is achieved by use of a brass aperture $100 \mathrm{~cm}$ downstream from the initial degrader. The dose distribution 
of the beam in a water phantom is measured by scanning in depth and traverse to the beam using a silicon diode (Koehler, 1967). The absolute dosimetry is established by an intercalibration of the diode with a Faraday cup (Koehler, 1967).

PATIENT IMMOBILISATION AND ALIGNMENT The patient's head was immobilised and held in position in a prototype head holder (Gragoudas et al., 1977). This allows controlled movement of the head in 3 orthogonal directions together with rotation about 2 orthogonal axes. It is thereby possible to bring the lesion to the centre of the axis of rotation and to make orthogonal adjustments of the lesion relative to the proton beam. A narrow light beam of $2 \mathrm{~mm}$ diameter coaxial with the central axis of the proton beam was used to position the tumour relative to the beam. The tumour margins were previously defined on the ocular surface by transillumination in relation to anatomical landmarks of the iris and conjunctiva. Immobilisation of the eye was achieved by voluntary fixation during treatment, which maintained precise alignment of the tumour and the beam (Fig. 6). Eye position was continuously monitored by a closed-circuit television monitor (Gragoudas et al., 1977); no more than $0.5 \mathrm{~mm}$ of motion was allowed. The patient received a total of 4750 rads given in 5 fractions over a period of 9 days.

\section{Follow-up}

The patient was examined after completion of the treatment, then after 1 month, and subsequently at 3-month intervals. No growth of the lesion has been observed after 21 months of follow-up, and signs of regression are evident. Alterations of the tumour pigmentation were observed 4 months after irradiation, and decrease in the height of the tumour was found on follow-up (Figs. 7, 8) and ultrasound examinations. A ${ }^{32} \mathrm{P}$ uptake test that was repeated 14 months after irradiation became negative at the $19 \%$ level. No ocular or extraocular complications have been observed during the follow-up period, and the patient has no complaints about her ocular and visual function or eye comfort.

\section{Discussion}

High-energy proton beams provide a compelling approach to localised ocular irradiation. These accelerated heavy particles have minimal scatter and can be collimated into narrow beams which retain their cross-sectional form during penetration. Their range is finite and energy-dependent, so that almost no radiation is delivered beyond the target.
Furthermore, they undergo maximal energy loss through ionisation as they stop, resulting in a characteristic maximum dose, called the Bragg peak, at the end of the beam path.

The precision and reliability of the aiming techniques for ocular irradiation have been shown previously in animals (Constable et al., 1975) and in man (Gragoudas et al., 1977). Fractionation of the dose, which increases the tolerance of the normal tissues and permits the delivery of higher doses to the tumour, is feasible with this method and has been used in the treatment of choroidal melanomas (Gragoudas et al., 1977). In addition, the possible presence of resistant hypoxic tumour cells renders fractionation of radiation advantageous. Although no increase in the lens opacities of the treated eye have been noted in our patient, cataract formation seems probable considering the dose used. However, it is likely that the opacities will be eccentric and not progressive, thus obviating the necessity of surgery (Stallard, 1968). Only further experience with a large number of treated cases can clarify this question. The limitations of iridocyclectomy and the problems encountered with other forms of localised irradiation make the use of the proton beam technique attractive for the treatment of ciliary body melanomas.

This investigation was supported by Public Health Service Research Grant 5-R01-CA-17638-02, from the National Cancer Institute. One of us (M. G.) is supported by a Research Career Development award CA-00251-01, also from the National Cancer Institute.

\section{References}

Constable, I. J., Koehler, A. M., and Schmidt, R. A. (1975). Proton irradiation of simulated ocular tumors. Investigative Ophthalmology, 14, 547-555.

Gragoudas, E. S., Goitein, M., Koehler, A., Verhey, L., Tepper, J., Suit, H., Brockhurst, R., and Constable, I. (1977). Proton irradiation of small choroidal malignant melanomas. American Journal of Ophthalmology, 83, 665-673.

Kjellberg, R. N., and Klinian, B. (1975). Bragg peak proton hypophysectomy for hypopituitarism, induced hypopituitarism and neoplasms. Progress in Neurologic Surgery, 6, 295-325.

Koehler, A. M. (1967). Dosimetry of proton beams using small silicon diodes. Radiation Research, 7, 53-63.

Linnic, L. F. (1968). Surgery of tumours of the ciliary body and base of the iris. British Journal of Ophthalmology, 52, 289-296.

Muller, H. K., Lond, O. E., Sölner, F., and Seidel, G. (1966). Die operative Behandlung von Tumoren des Kammerwinkels und des Ciliarkörpers. Documenta Ophthalmologica, 20, 500-508.

Palm, E., and Linder, B. (1968). Excision of tumours in the iris and the ciliary body. Acta Ophthalmologica, 46, 521528.

Reese, A. B., Jones, J. S., and Cooper, W. C. (1968). Surgery 
for tumours of the iris and ciliary body. American Journal of Ophthalmology, 66, 173-184.

Stallard, H. B. (1964). Partial cyclectomy: Some further modifications in technique. British Journal of Ophthalmology, 48, 1-6.

Stallard, H. B. (1968). Malignant melanoblastoma of the choroid. Modern Problems in Ophthalmology, 7, 16-38. Stallard, H. B. (1973). Eye Surgery, p. 510. Williams \& Wilkins: Baltimore.

Vail, D. T. (1971). Iridocyclectomy. A review: gleanings from the literature. American Journal of Ophthalmology, 71 (Suppl.), 161-168. 